\title{
ROMANIAN
}

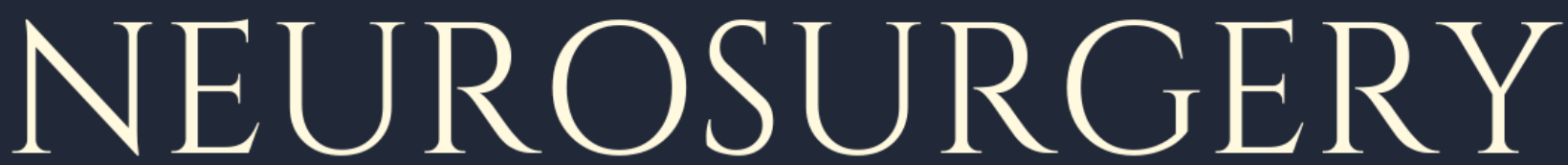

Vol. XXXIV | No. 4 December 2020

Surgical management of Rolandic area meningioma in the era of intraoperative neurophysiological monitoring

\author{
Mihaela Coșman, \\ Ionuț Mihail Panțiru, \\ Andrei lonuț Cucu, \\ Andreea Lenuța Atomei, \\ Gabriela Florența Dumitrecu, \\ Ion Poeată
}




\section{Surgical management of Rolandic area meningioma in the era of intraoperative neurophysiological monitoring}

\section{Mihaela Coșman ${ }^{1}$, Ionuț Mihail Panțiru², Andrei Ionuț $\mathrm{CuCu}^{2}$, Andreea Lenuța Atomei ${ }^{3}$, Gabriela Florența Dumitrecu ${ }^{4}$, Ion Poeată ${ }^{5}$}

\author{
1 Department of Neurosurgery. Emergency County Hospital, Braila, \\ ROMANIA \\ 2 Department of Neurosurgery. "N. Oblu" Emergency Clinical \\ Hospital, Iași, RomANIA \\ 3 6th-year student. Gr. T. Popa University of Medicine and Pharmacy, \\ Iași, ROMANIA \\ 4 Department of Anatomopathology. "N. Oblu" Emergency Clinical \\ Hospital, Iași, ROMANIA \\ 5 Department of Neurosurgery. Gr. T. Popa University of Medicine \\ and Pharmacy, Iași, ROMANIA
}

\begin{abstract}
Introduction. The advantages and the necessity of intraoperative neurophysiological monitoring (IOM) in the surgery of motor area infiltrative tumours is well known. The use of this technique for Rolandic meningioma is still debatable. The absence or the loss of the cleavage plan and an infiltrative border make the dissection exceedingly difficult and increase the risk of new postoperative motor disfunction.
\end{abstract}

Materials and methods. We evaluated the impact of IOM, especially direct cortical stimulation on the degree of resection, new postoperative deficits, symptom remission and clinical-imagistic aspects at one-year follow up of 19 cases of Rolandic meningioma admitted in Third Department of Neurosurgery," Prof. Dr N. Oblu" Emergency Clinical Hospital, Yassi, Romania, between January 2014 and July 2018.

Results. More than half of the cases $(57,88 \%)$ had epileptic manifestations as the main clinical symptom with the Jacksonian seizures being on the first place $(31,57 \%)$, followed by progressive paresis $(26,31 \%)$ and other nonspecific symptoms. Intraparenchymal preoperative oedema was observed in $36,84 \%$ of patients. The intensity of direct cortical stimulation was between 6-13 $\mathrm{mA}$ (median $=9 \mathrm{~mA}$; mode = $12 \mathrm{~mA}$ ). Simpson degree of resection was dominated by $\mathrm{S3}-47,36 \%$ and $\mathrm{S} 4$ was obtained in $15,78 \%$ of cases. Postoperative the outcome was favourable for $73,68 \%$ patients with 5,26\% motor aggravation and 10,52\% new deficits. At one-year follow up no imagistic recurrence was observed and the permanent motor deficit was maintained in one of the three cases (5,26\%).

Conclusion. Even though meningiomas are extranevraxial lesions and those located on the convexity have a low risk of complication, the absence of a clear dissection plan between the tumour and the adjacent motor cortex is associated with a high risk for new postoperative neurological deficits. Therefore, it is important to perform

\section{Keywords \\ Rolandic area, \\ intraoperative \\ neurophysiological \\ monitoring, \\ meningioma, \\ tumour resection, \\ motor cortex, \\ cortical mapping}

$\triangle$

Corresponding author: lonuț Mihail Panțiru

"N. Oblu" Emergency Clinical Hospital, Iași, Romania

ionut.mihail26@yahoo.com

Copyright and usage. This is an Open Access article, distributed under the terms of the Creative commons Attribution Non-Commercial No Commons Attribution Non-Commercial No
Derivatives License (https://creativecommons org/licenses/by-nc-nd/4.0/) which permits noncommercial re-use, distribution, and reproduction in any medium, provided the original work is unaltered and is properly cited.

The written permission of the Romanian Society of Neurosurgery must be obtained for commercial re-use or in order to create a derivative work.

ISSN online 2344-4959

(C) Romanian Society of Neurosurgery

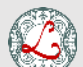

First published

ondon Academic Publishing wwww.lapub.co.uk 
cortical mapping for Rolandic meningioma, to determine the location of the primary motor area and to protect it from mechanical and vascular trauma, during tumour resection.

\section{INTRODUCTION}

Resection of lesion located in eloquent areas e.g., primary motor area is associated with an increased risk of postoperatively neurologic deficits. For a good outcome it is mandatory, for us, to know the location of this area and to protect it from mechanical and vascular intraoperative injures. The landmarks offered by the preoperative radiological images are of great help, but not sufficient $[10,35]$. The continuous development of medical technology comes in our aid to perform maximal resection with minimal motor dysfunction, knowing the impact on overall survival and on the progression free survival $[8,29,32]$.

On this line, intraoperative neurophysiological monitoring (IOM) which includes mapping procedures (direct / subcortical stimulation) and monitoring technique (evoked potentials) offers a real time feedback and helps to establish the location of the functional area from the operative field. If the use of IOM is clear for infiltrative lesions like gliomas, the necessity of it in cases of meningiomas remains questionable [2,21,31].

One functional area in which this lesion may appear is the central gyrus region. The definition of Rolandic meningioma is represented by the direct anatomical contact, observed on T2 Weighted magnetic resonance imaging (T2W-MRI) of the tumour with the precentral and postcentral gyrus [25]. The presence of the cleavage plan around the eloquent brain help protect it during resection. When this landmark is lost or its not present mechanical trauma and vascular alterations over the cortex may generate new motor deficits. An intraoperative evaluation of the surrounding brain with the enhancement of the primary motor area is important, with the purpose of maximal resection with minimal neurological dysfunction. This goal is desirable especially when the most common symptoms of presentation are the epileptic seizures and the nature of the lesion is with good prognosis $[6,21,31]$.

We proposed in this article to present the impact of using IOM in meningioma surgery located in central gyrus region regarding the clinical and radiological postoperative evolution first day after surgery and at one year follow up period along with a review of the literature.

\section{MATERIALS AND METHODS}

The study group included patients with meningioma located in central region, diagnosed using contrast enhancement head MRI imaging, admitted in the 3rd neurosurgery department of 'Prof. Dr N. Oblu' Clinical Emergency Hospital Iasi, between 1 January 2015 and 1 July 2018, who underwent surgical resection. Inclusion criteria in the study group: meningioma located in central gyrus region, imagistic diagnosed and histologically confirmed; age over 18 years; intraoperative use of IOM; consent to be included in the study. Exclusion criteria from the study group: other histological tumour subtypes localized in central region; cases to which conservative management was performed; patients with pacemaker; patients who failed to come for their one-year follow-up examination and incomplete cases data.

This technique was performed using the Nim Eclipse device from Medtronic. Direct cortical / subcortical stimulation was achieved by means of the short-train technique or train of five. The parameters used were: $3 \mathrm{~Hz}$, interstimulus interval = 4 mseconds, length $=500 \mu$ seconds. The intensity was included in the interval $6-13 \mathrm{~mA}$. The recording electrodes were placed in abductor pollicis brevis muscle (m.), biceps brachii m., orbicularis orris and oculi m., tibialis anterior m. and abductor hallucis m, depending on tumour precise location.

\section{RESULTS}

76 patients with various histological tumour types were initially enrolled in the study group, but 6 of them were excluded because they did not come to the one-year follow-up examination after surgery and 4 were excluded because they had a pacemaker and we could not perform IOM. In the end, the group included 19 cases of meningioma with Rolandic location. The age distribution interval was between 25 - 73 years with a female dominance $-52,63 \%$ of all the cases. Most frequent symptom was Jacksonian seizure (31,57\%), followed by motor deficit - progressive brachial/ crural paresis $(26,31 \%)$, partial seizures $(15,73 \%)$ and nonspecific manifestation like headache, intracranial hypertension and grand mall seizure (each 10,52\%). 
From the anatomical point of view $47,36 \%$ ( 9 cases) were located on the convexity, $47,36 \%$ were parasagittal and $5,26 \%$ (one case) had the insertion of the falx cerebri. 5,26\% of the patients underwent preoperative embolization. Intraparenchymal oedema was observed in T2 / FLAIR MRI sequences in a proportion of $36,84 \%$ ( 7 cases). An illustrative is presented in Figure 1.
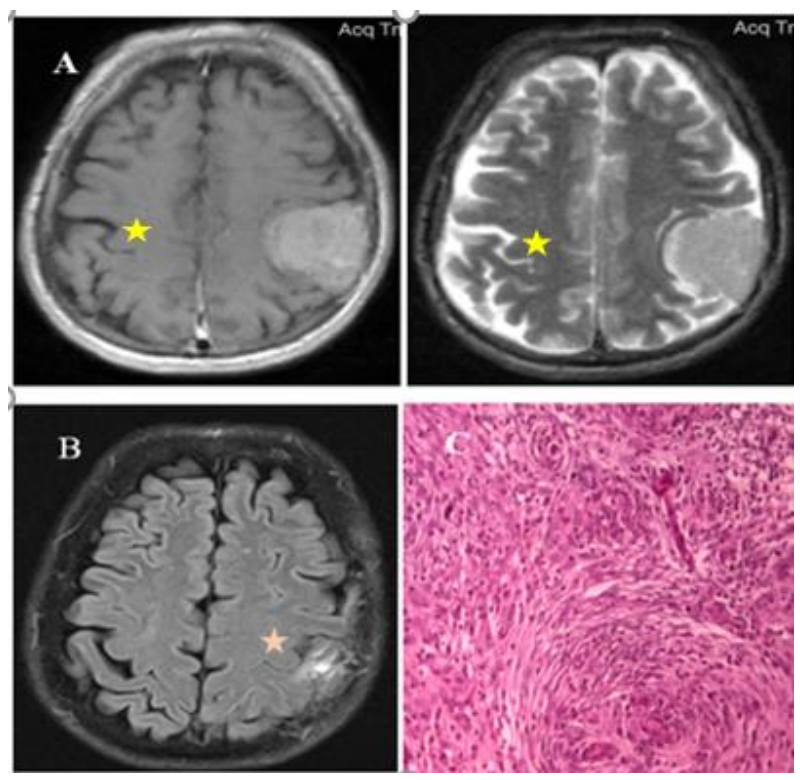

Figure 1. Clinical case: female, 48 years, Jacksonian seizures, no motor deficit, Rolandic lesion. (A) preoperative MRI (T1WI with enhancement and $\mathrm{T} 2 \mathrm{WI}$ ), yellow star = contralateral primary motor area (omega sign), no clear demarcation of the ipsilateral motor area, no perilesional oedema. (B) one-year postoperative follow-up MRI (T2 dark-fluid), pink star=reshaping of the motor area with normal morphology. (C) Meningioma grade II (WHO classification): densely cellularized lesion with meningothelial cells arranged in wide cords. (Hemalaun-Eozina staining, X20).

According to World Health Organisation (WHO) the histological result was classified as WHO grad I: $84,21 \%$ and WHO grade II: $15,78 \%$. Simpson degree of resection was: S1 - 2 patients $(10,52 \%)$, S2 - 5 patients $(26,31 \%)$, S3- 9 cases $(47,36 \%)$ and S4 - 3 cases $(15,78 \%)$. The intensity of direct cortical stimulation was between 6-13 mA, with the following statistical parameters: median $=9 \mathrm{~mA}$ and the mode $=12 \mathrm{~mA}$. Postoperative the outcome was favourable with symptoms resolution for $73,68 \%$ cases, stationary for $10,52 \%$ cases and with functional alteration for $15,78 \%$ cases ( 2 patients presented new neurologic deficit and one aggravated the initial motor disfunction).
One-year evaluation reveal favourable outcome in $89,47 \%$ and stationary in $10,52 \%$. From the three cases with postoperative motor disfunction, in 5,26\% of them persisted the deficit. No recurrence was observed on the control MRI and no tumour growth on the $15,78 \%$ of Simpson IV cases.

\section{Discussions}

Meningioma represent the most common benign primary brain tumour with an increasing incidence, being more frequent in elderly and in women $[1,11,16]$. Usually between the brain and the lesion there is a cleavage plan which allows a safe resection. When this plan is lost or it does not exist, vascular impairment and mechanical trauma of the brain tissue may be a consequence of tumour ablation. This is very important when the perilesional tissue is represented by functional areas, e.g., central gyrus region, hence the higher postoperatively motor deficit and an increased rate of complication for Rolandic meningioma compared with other convexity meningioma $[3,9,18,23]$.

Perioperatively imaging techniques characterizes the relationship between the tumour and the eloquent cortex. Standard MRI, functional MRI, 3D tractography and neuronavigation system are important tools regarding the resection of lesions located in eloquent cortex, planning the approach and guiding further tumour ablation $[20,22,27,30]$.

In some meningioma cases, the landmarks may not correspond intraoperatively because of the displacement of the normal anatomy induced by the tumour growth. In others the cleavage plan may be lost near the central gyrus region and the arachnoid may be invaded [5]. To avoid such situation where the dissection is difficult to realise without harming the surrounding cortex intraoperative neurophysiological monitoring is being used for a real time functional feedback. In a study from 2019 published by Raffa et al., it is evaluated even the advantage of combining the navigated transcranial magnetic stimulation with IOM for detection of the presence or the absence of the arachnoid cleavage plan and the functional tissue. The correspondence with the IOM results was in a percentage of $94,2 \%$ [28]. With all those tools available, in the literature there is still a controversy about the techniques association and intraoperative necessity of neurophysiological monitoring for all meningioma $[25,26,31]$. 
From the anatomical point of view Rolandic meningioma compresses convexity meningioma, falx, falx-sinus lesions of middle one third of the sagittal sinus in contact with precentral and postcentral gyrus and sinus. Beside the surrounding functional areas, the vascular representation is of the same importance e.g., Rolandic draining vein [6]. Because these lesions have a tendency of being smaller, the difficulty of the intervention can be underestimated and so may be associated with higher complication [25].

Motor area meningioma need special anaesthesia protocols when IOM is used, the aim being to avoid the medication that interacts with muscle relaxation. Synthetic opioids such as Fentanyl and sedative-hypnotic agents (Propofol) are preferred when cortical stimulation or evoked potentials are recorded, since those drugs can maintain a constant serum concentration with insignificant effect over motor response registration $[14,15,24]$.

Usually, one of the most common presentation symptoms is represented by epileptic seizure $(47,6 \%$ - Ostry et al.,2012; 38,46\% - Bi et al., 2013; 42\% - Deng et al.,2014) $[4,6,25]$. In our study this manifestation occurred in $57,88 \%$ of patients, with a dominance of Jacksonian seizures $(31,57 \%)$. Especially for these patients with no motor deficit preoperatively it is important to determine the relation between the meningioma and the surrounding brain tissue.

The dominance of this clinical presentation is explained by the tumour mechanism of action. Being an extranevraxial lesion, it compresses the brain tissue inducing hemodynamical changes at the level of myelin, oxygenation and intracellular water. This is important because there is not a real loss of neurons and explains the remission of the symptoms after resection. Even the patients with preoperative motor deficits may improve the muscle strength postoperatively [17]. Though the meningioma is completely resected in some patients the outcome after the operation is stationary. The persistence of the seizures after the surgery is explained, in some cases by the cerebral changes induced by a slow-growing tumour with the appearance of epileptogenic foci e.g., hippocampal scleroses and cortical dysgenesis, beside local microenvironment abnormal discharges. In these cases, it seems that only the removal of the central gyrus region tumour is not enough [7,33]. In a study published by Deng et al., in 2014 from all 26 patients presented with epilepsy, after tumour resection 88 , $46 \%$ were seizures free at mean follow up of 16 months under antiepileptic drug medication and one patient had seizures recurrence in less than 6 months postoperatively [6]. In our study, after symptoms remission the patient did not report any new seizure at the one-year follow up or recurrence.

When there is pial adhesions, brain invasion or irregular borders intracapsular resection is recommended to preserve the functional cortex which is usually identified by direct cortical stimulation (e.g., monopolar anodic stimulation Ostry et al.,2012) [25]. In our study we performed brain mapping before starting to remove the meningioma to identify the primary motor area. Usually, this aria was modified being push by the tumour growth and surrounded the lesion. The intensity interval was between $6-13 \mathrm{~mA}$, the most frequent threshold value which generated motor response was $13 \mathrm{~mA}$. The cortical stimulation was repeated every time when the junction brain-tumour was reached, to ashore that further dissection does not affect the eloquent cortex. Intermittent minimal traction was applied.

The surgical aim is symptom remission and if it is the case to leave the smallest cortical layer of tumour to prevent neurological alteration, even though this means subtotal resection - Simpson IV $[12,19]$. On the one hand this approach is preferable because of the slow growth pattern of the lesion and because gross resection is associated with increased morbidity for the infiltrative borders type, on the other hand some studies found that Simpson resection grade is a predictor factor for recurrence. In this condition the intraoperative decision is tailored depending on the particularities of the case $[13,36]$.

In our study Simpson IV was obtained at $15,74 \%$ of patients, all of them were located parasagittal with no cleavage plan and with positive stimulation response surrounding the tumour. Other characteristic of those three cases was the fact that it was observed an important venous component involvement, with the Rolandic vein being encased in tumour capsule and the superior sagittal sinus being partially obstructed. In other publication various result of subtotal resection were presented from $26,2 \%$ (Ostry et al., 2012) to 1,1\% (Ottenhause et a., 2018) $[25,26]$. Ostry et al., mentioned that in some 
patients the remanent tumour was not even detected on the postoperative MRI, the estimated volume being of $0,1 \mathrm{~cm}^{3}$ and the Simpson IV grade of the case was based on surgeons' report. Usually, the residual layer was less than $0,5 \mathrm{~cm}^{3}$ [25]. Even though Simpson grad IV was present in our result one the first place from the point of view of resection was Simpson grade II (47,36\%) matching with the literature results.

It is important to keep in mind that clinical presentation with preoperative muscular strength dysfunction may alter the stimulation response, decreasing the technique accuracy. From our patients $26,31 \%$ presented progressive paresis and the intensity used to generate motor response was the highest from the study group. An aggressive traction and dissection, when the cleavage plan is lost, is associated postoperatively with a higher risk for motor deficit [17,34]. Ostry et al., in a study from 2012 observed that the difference of the threshold value between the direct cortical stimulation and the value obtained after stimulating through the mass lesion was $\leq 2 \mathrm{~mA}$ has an impact over outcome. In this situation he stopped the resection even though it was intracapsular, to prevent new motor deficits. When the motor evoked potentials are used, the need for an increased threshold to generate response is a warning signal [25].

How is to be expected, the motor preservation is the main goal in surgical resection of Rolandic meningioma. Starting from this idea some authors studied the prognostic factors for the risk of motor impairment (aggravation of the symptoms or new ones). Ottenhausen et al., found that a high rate of neurological deficit was associated with parafalcine insertion, large tumoral mass and perilesional oedema. Another negative prognostic factor was found to be the necessity for preoperative embolization and the involvement of the Rolandic drainage vein [26]. From our group of patients in one case it was necessary to perform an endovascular procedure, tumour's location being on the falx cerebri to facilitate the resection.

Progressive paresis as an admitting symptom beside disturbing the IOM is considered to be a negative prognostic factor for the motor outcome (47, $2 \%$ vs $22,2 \%, p=0,017$, Ottenhausen et al., 2018) [26]. In our group of patients in one case we had aggravation of the pre-existent deficit and new deficit was observed in $10,52 \%$ of the cases. The image control showed central gyrus region oedema and no haemorrhage.

Other values from the literature regarding the new motor deficit range from 7,69\% (Lee et al., 2016) to $34,61 \%$ (Bi et al., 2013) [4,17]. The latter reference presents the results from 26 parasagittal central region meningioma microsurgical resected. No Simpson grade IV was found, only grade I $(30,8 \%)$, grade II $(46,2 \%)$ and grade III $(23,1 \%)$ but as mentioned the aggravation was observed in one third of the cases. Therefor complete tumor removal was associate with a higher negative outcome. Another important fact is that the authors do not report the use of cortical stimulation or evoked potential generation [4].

The main postoperative outcome of our patients was favourable with symptom remission in $73,68 \%$. Almost the same percentage was obtained and by Lee at al., in $2016-76,92 \%$. The first place for clinical evolution is maintained and with $60,3 \%$ (Ottenhausen et al., 2018), 65,39\% (Bi et al., 2013) and the high clinical amelioration was $81 \%$ (Ostry et al., 2012) $[4,17,25,26]$.

An important remark it that at one year follow up period just one patient of the three with postoperatively aggravated or new deficit maintained the motor disfunction. Practically the permanent neurologic deficit for our group was $5,26 \%$. Overall, the neurological outcome was favourable in $89,47 \%$ of the cases, no imagistic recurrence was observed on the control MRI but further follow up must be done.

\section{CONCLUSIONS}

Rolandic meningiomas, even though are extranevraxial lesions represents a challenge from the surgical point of view. A careful dissection is mandatory, the venous drainage must be preserved and the surrounding functional tissue must be protected from mechanical trauma. When the cleavage plan is lost precentral and postcentral cortex may be affected and secondary after the resection new motor deficits may appear. To prevent unwanted cortical damage intraoperative neurophysiological monitoring is used and if necessary, a thin layer of meningioma is left over the cortex. 


\section{DisClOSURES}

The authors report no conflict of interest concerning the materials or methods used in this study or the findings specified in this paper.

\section{REFERENCES}

1. Achey RL, Gittleman H, Schroer J et al. Non-malignant and malignant meningioma incidence and survival in the elderly from 2005-2015 using the Central Brain Tumor Registry of the United States. Neuro Oncol, 21(3):380-391, 2019.

2. Bander DE, Shelkov E, Modik O et al. Use of the train-offive bipolar technique to provide reliable, spatially accurate motor cortex identification in asleep patients. Neurosurg Focus, 48: 1-6, 2020.

3. Berg-Johnes J, Hogestol EA. Supplementary motor area syndrome after surgery for parasagittal meningiomas. Acta Neurochir (Wien), 160(3):583-587, 2018.

4. Bi N, XU RX, Liu RY et al. Microsurgical treatment for parasagittal meningioma in the central gyrus region. Oncol Lett 6: 781-784, 2013.

5. Cimino PJ. Malignant progression to anaplastic meningioma: Neuropathology, molecular pathology, and experimental models. Exp Mol Pathol, 99(2):354-9, 2015.

6. Deng WS, Zhou XY, Li ZJ et al. Microsurgical treatment for central gyrus region meningioma with epilepsy as primary symptom. J Craniofac surg, 25: 1773-1775, 2014.

7. Dhiman V, Rao S, Sinha $S$ et al. Outcome of lesionectomy in medically refractory epilepsy due tonon-mesial temporal sclerosis (non-MTS) lesions. Clin Neurol Neurosurg, 115(12):2445-53, 2013.

8. Duffau H. Diffuse low-grade glioma, oncological outcome and quality of life: a surgical perspective. Curr Opin Oncol, 30:383-389, 2018.

9. Elzarief AA, Ibrahim MF. Long-term follow-up of motor function deterioration following microsurgical resection of middle third parasagittal and falx meningioma. Egypt J Neurol Psychiatr Neurosurg, 54(1):9, 2018.

10. Giamouriadis A, Lavrador JP, Bhangoo R et al. How many patients require brain mapping in an adult neurooncology service? Neurosurg Rev, 43:729-738, 2020.

11. Holleczek B, Zampella D, Urschart S et al. Incidence, mortality and outcome of meningiomas: A populationbased study from Germany. Cancer Epidemiol, 62:101562, 2019.

12. Hou W, Ma Y, Xing H, Yin Y. Imaging characteristics and surgical treatment of invasive meningioma. Oncol Letters, 13: 2965-2970, 2017.

13. Hwang WL, Marciscano AE, Niemierko A et al. Imaging and extent of surgical resection predict risk of meningioma recurrence better than WHO histopathological grade. Neuro Oncol, 0:1-10, 2015.

14. Isik B, Turan G, Abitagaoglu S et al. A comparison of the effects of desflurane and total intravenous anaesthesia on the motor evoked responses in scoliosis surgery. Int J Res Med Sci,5(3):1015-1020, 2017.

15. Kawaguchi $\mathrm{M}$, lida $\mathrm{H}$, Tanaka $\mathrm{S}$ et al. A practical guide for anesthetic management during intraoperative motor evoked potential monitoring. J Anesth, 34(1):5-28, 2020.

16. Ko CC, Chen TY, Lim SW et al. Prediction of Recurrence in Parasagittal and Parafalcine Meningiomas: Added Value of Diffusion-Weighted Magnetic Resonance Imaging. World Neurosurg, Worl Neurosurg, 124: e470e479, 2019.

17. Lee Sj, Hwang SC, Im SB, Kim BT. Surgical resection of non-glial tumors in the motor cortex. Brain Tumor Res Treat, 4 (2):70-76, 2016.

18. Lemee JM, Corniola MV, Broi MD et al. Early Postoperative Complications in Meningioma: Predictive Factors and Impact on Outcome. World Neurosurg, 128: e851-e858, 2019.

19. Lin Q, Ling F, Xu G. Invasive benign meningioma: Clinical characteristics, surgical strategies and outcomes from a single neurosurgical institute. Exp Ther Med, 11(6):2537-2540, 2016.

20. Low D, Lee CK, Dip LLT et al. Augmented reality neurosurgical planning and navigation for surgical excision of parasagittal, falcine and convexity meningiomas. Br J Neurosurg, 24(1):69-74, 2010.

21. Magill ST, Han SJ, Li J et al. Resection of primary motor cortex tumors: feasibility and surgical outcomes. J Neurosurg, 129:961-972, 2018.

22. Morin O, Chen WC, Nassiri F et al. Integrated models incorporating radiologic and radiomic features predict meningioma grade, local failure, and overall survival. Neurooncol Adv, 1(1):011, 2019.

23. Nanda A, Bir SC, Konar S, Maiti TK, Bollam P, World Health Organization Grade I Convexity Meningiomas: Study on Outcomes, Complications and Recurrence Rates, World Neurosurg, 89:620-627.e2, 2016.

24. Nunes RR, Bersot CDA, Garritano JG. Intraoperative neurophysiological monitoring in neuroanesthesia. Curr Opin Anesthesiol, 31:532-538, 2018.

25. Ostry S, Netuka D, Beneš V. Rolandic area meningioma resection controlled and guided by intraoperative cortical mapping. Acta Neurochir (Wien), 154:843-53, 2013.

26. Ottenhausen M, Rumalla K, Younus I et al. Predictors of postoperative motor function in rolandic meningiomas. J Neurosurg, 1:1-6, 2018.

27. Panesar SS, Abhinav K, Yeh FC et al. Tractography for surgical neuro-oncology planning: towards a gold standard. Neurotherapeutics, 16: 36-51, 2019.

28. Raffa G, Picht T, Scibilia A et al. Surgical treatment of meningiomas located in the rolandic area: the role of navigated transcranial magnetic stimulation for preoperative planning, surgical strategy, and prediction of arachnoidal cleavage and motor outcome. J Neurosurg, 14;1-12, 2019.

29. Ritaccio AL, Brunner P, Schalk G. Electrical stimulation 
mapping of the brain: basic principles and emerging alternatives. J Clin Neurophysiol, 35: 86-97, 2018.

30. Romero-Garcia R, Erez $\mathrm{Y}$, Oliver $\mathrm{G}$ et al. Practical application of networks in neurosurgery: combined 3D printing, neuronavigation, and pre-operative surgical planning. World Neurosurg, 137:1-22, 2020.

31. Rossi M, Nibali MC, Vigano L et al. Resection of tumors within the primary motor cortex using high-frequency stimulation: oncological and functional efficiency of this versatile approach based on clinical conditions. J Neurosurg, 9:1-13, 2019.

32. Sala F. Penfield's stimulation for direct cortical motor mapping: An outdated technique? Clin Neurophysiol, 129:2635-2637, 2018.
33. Spencer S, Huh L. outcome of epilepsy surgery in adults and children. Lancet Neurol 7: 525-37, 2008.

34. Tang $\mathrm{H}, \mathrm{Xu} \mathrm{F}$, Lin L et al. Intra-operative motor function preservation for resection of primary motor cortex meningioma. Transl Cancer Res, 7(6):1666-1674, 2018.

35. Thon N, Tonn JC, Kreth FW. The surgical perspective in precision treatment of diffuse gliomas. Onco Targets Ther,12: 1497-1508, 2019.

36. Winther $\mathrm{WL}$, Torp SH. The significance of the extent of resection in modern neurosurgical practice of WHO grade I meningiomas. World Neurosurg, 99:104-110, 2017. 